\title{
Théologiques
}

\section{Le discernement spirituel, pivot de la vie religieuse}

\section{Fabrizio Vecoli}

Volume 22, numéro 2, 2014

Le discernement spirituel

URI : https://id.erudit.org/iderudit/1035684ar

DOI : https://doi.org/10.7202/1035684ar

Aller au sommaire du numéro

Éditeur(s)

Faculté de théologie et de sciences des religions, Université de Montréal

ISSN

1188-7109 (imprimé)

1492-1413 (numérique)

Découvrir la revue

Citer ce document

Vecoli, F. (2014). Le discernement spirituel, pivot de la vie religieuse.

Théologiques, 22(2), 5-15. https://doi.org/10.7202/1035684ar d'utilisation que vous pouvez consulter en ligne.

https://apropos.erudit.org/fr/usagers/politique-dutilisation/ 


\title{
Le discernement spirituel, pivot de la vie religieuse
}

\author{
Fabrizio VeCOLI* \\ Histoire du christianisme \\ Université de Montréal (Canada)
}

Le discernement, dans la tradition chrétienne, est le pouvoir cognitif par excellence, l'hypostase charismatique de la prétention au monopole exclusif sur la vérité de la part du spécialiste du sacré. Discerner signifie en premier lieu séparer la vérité du mensonge, et par suite affirmer une vérité définitive et exclusive, une exigence perçue et vécue de façon tout à fait originale par la pensée occidentale le long de son développement pluriséculaire. La détermination de la vérité au-delà du conflit des interprétations est une revendication qui remonte à l'antiquité religieuse, notamment judéo-chrétienne (Assmann 1997). Le discernement, comme terme et comme concept, fait son apparition - quant à lui — dans les épîtres pauliniennes. Toutefois, sa première élaboration a lieu dans le cadre de la doctrine monastique ancienne (Vecoli 2006; Rich 2007); il devient par la suite une notion fondamentale de la théologie post-tridentine, en particulier dans le cadre de la direction spirituelle (Ignace de Loyola, par exemple) et dans la théorie du gouvernement de la communauté religieuse (CopelandMachielsen 2013). Mais l'étendue de ses applications est bien plus vaste qu'on ne le penserait de prime abord, car il intervient à chaque fois que l'inspiration divine provoque une prise de position en tension ou en désaccord avec la logique profane. Il agit particulièrement là où le spécialiste du

* Fabrizio Vecoli est professeur adjoint à la Faculté de théologie et sciences des religions de l'Université de Montréal. Son travail de recherche se concentre actuellement sur le concept de discernement dans l'histoire du christianisme. Parmi ses œuvres, la monographie (2007) Il sole e il fango. Puro e impuro tra i Padri del deserto, Rome, Edizioni di Storia e Letteratura reflète son intérêt pour l'histoire du monachisme ancien. 
sacré doit délibérer en dehors du tracé des règles établies, soit que celles-ci ne s'appliquent pas, soit qu'elles n'existent pas. Aux yeux du profane, il apparaît comme la dimension arbitraire d'une autorité religieuse. Ce que l'on constate dans le christianisme, c'est que l'accumulation inédite des multiples fonctions socioreligieuses liées à ce "don divin" peut être comprise comme la traduction dans la praxis religieuse d'une revendication théologique typiquement monothéiste.

Le but de ce numéro de Théologiques est d'étudier le thème du discernement sans établir des bornes préalables à l'éventail des disciplines susceptibles de l'appréhender. C'est pour cela qu'on y trouvera des études autant textuelles qu'historiques, anthropologiques, théologiques ou d'histoire de la pensée. Non seulement l'approche multidisciplinaire (à défaut de pouvoir être, à ce stade, interdisciplinaire) s'avère féconde envers tout objet d'étude, mais elle l'est d'autant plus en ce qui a trait à celui-ci en particulier. En effet, le discernement spirituel se situe au croisement de plusieurs dimensions de la vie religieuse, car il est à la fois: un engrenage essentiel de la théorie ascétique, une source d'autorité dans le gouvernement de la communauté de foi, l'instrument de contrôle de l'effervescence mystique, la légitimation d'une ecclésiologie collégiale en réformatrice, le pivot d'une pratique visant à éclaircir une vocation religieuse, le critère de cohérence dans les spécificités de la direction spirituelle, et ainsi de suite. Il s'ensuit que l'exercice de cette qualité particulière de l'esprit a des répercussions significatives sur les plans théologique, social, politique (et autres encore). Aucune discipline ne peut espérer couvrir à elle seule les maintes ramifications (dans la pensée, dans la pratique, dans l'organisation institutionnelle ou sociale, etc.) d'un concept comme celui-ci. Les articles présentés ici ouvrent donc à cette pluralité scientifique.

Afin que le lecteur s'y retrouve, il est sans doute utile de fournir quelques repères sur l'origine et la nature du discernement dans la tradition chrétienne - sur laquelle portent la majorité des articles. Ce que l'on nomme "discernement ", à la suite de la tradition monastique chrétienne, est à l'origine le "discernement des esprits" (diakrisis ton pneumaton en grec, discretio spirituum en latin), un charisme (charisma en grec, gratia en latin) divin dont la première attestation se trouve dans les épîtres de Paul (1Co 12,10). La tradition successive définira ce pouvoir comme - avant toute autre chose - la capacité de distinguer les visions reçues de Dieu de celles envoyées par Satan. Dans la logique de l'élaboration théologique, les auteurs chrétiens partent du présupposé que l'homme de Dieu, syntonisé sur les fréquences métaphysiques du réel, se voit octroyer un surplus de 
connaissance par rapport à ses semblables profanes. De cette circonstance découle une question vitale: s'agit-il d'une illusion - fruit de tromperies démoniaques ou de phantasmes internes à l'esprit humain — ou alors cette connaissance donne-t-elle réellement accès à la vérité dissimulée au-delà du voile du monde matériel ? Le discernement, selon la doctrine de ses théoriciens, résout ce doute et départage toute inspiration surnaturelle entre méprise et illumination. Il tranche là où aucun instrument profane n'est en mesure de le faire. Pour cela, on l'a parfois défini comme le «charisme de clairvoyance " (charisma dioratikon en grec) ou la "vue d'en haut" (anablepsis en grec). La capacité à discerner la bonne inspiration parmi les différents mouvements de l'esprit finit par s'identifier à un don de perspicacité d'origine divine.

Suivant l'accumulation de ses multiples fonctions au fil des siècles et des contextes, le discernement devient l'instrument qui préside à tout jugement et qui permet donc de répondre à toute question: le malade demande que faire pour obtenir la guérison, le paysan quand semer pour obtenir une bonne récolte; le magistrat comment juger d'une cause particulièrement compliquée, le gouverneur militaire s'il doit ou non entreprendre une campagne militaire, le novice quel régime ascétique lui conviendra le mieux pour atteindre le salut, l'évêque où se situe l'orthodoxie dans le foisonnement des courants doctrinaux, et ainsi de suite. Tous s'adressent au «saint» - le détenteur par excellence de ce don surnaturel — afin d'obtenir la réponse ultime à leurs questions. Celui auquel on reconnaît le pouvoir de discerner sera donc en mesure de lire dans le cœur des hommes, de fixer son regard dans les mystères célestes, de prévoir à l'avance les événements politiques ou naturels, de reconnaître la vraie doctrine parmi les hérésies, de juger toute situation tant sur le plan éthique que celui pratique.

$\mathrm{Au}$ fond, ce qu'on attend de l'autorité qui possède le discernement, c'est d'être en mesure de distinguer les mécanismes mystiques qui opèrent dans la structure de la réalité, mécanismes qui sont les fondements d'un réel inaccessible à la science profane. Outil gnoséologique multifonctionnel, cette faculté devient le pivot sur lequel s'appuie la religion dans son rapport - qui se veut le seul authentique — au monde.

Comme directeur de ce numéro, et dans la perspective d'histoire des religions où je me situe, je problématise la question que nous pose le discernement de la manière suivante. Quand une religion prétend affirmer une interprétation du réel qui lui est propre et qui se veut exclusive, ou du moins différente par rapport aux références ambiantes, elle se doit de s'appuyer sur un fondement capable de résister à la force corrosive des 
autres interprétations: qui peut décider de la perspective à adopter? Comment sortir du conflit des interprétations? Le discernement devient le fondement - infondé (à tout le moins selon des critères empiriques) d'une vérité contre les autres. Il intervient là où l'interprétation donnée n'est pas immédiate et ne va pas de soi: le «don de l'Esprit » opère donc dans un contexte conflictuel.

Les études sur le discernement montrent clairement qu'il s'agit d'un objet récurrent à l'intérieur de l'histoire du christianisme. En effet, il réapparait régulièrement à toutes les époques, expression de l'incontournable nécessité d'une connaissance fondatrice de cet édifice complexe qu'est la religion. Certes, il change selon les périodes et les contextes, mais sans jamais évacuer les élaborations précédentes: le long de ses déclinaisons historiques, il unit nouveauté de situation et intertextualité du concept, chaque fois récupérant la tradition en l'élaborant - donc en l'innovant - selon les exigences contingentes. Cette caractéristique de l'objet justifie une appréhension diachronique (c'est-à-dire une étude sur la période longue), qui se trouve représentée en ce numéro monographique: il a été demandé à des auteurs s'intéressant à des périodes différentes de traiter du discernement dans le monde spécifique de leur compétence afin de faire ressortir ce jeu complexe - toujours inextricable pour le théoricien, et pourtant visible au chercheur - entre «sameness » et "difference », entre spécificité (idiographique) et récurrence (nomothétique).

Il s'agit, au fond, d'un enjeu typiquement comparatif. Qu'y a-t-il de semblable ou de différent entre le discernement d'un moine du Iv siècle et celui d'une collégialité ecclésiale réunie au concile Vatican II ? Sommesnous autorisés à le considérer comme un seul et même objet? Les enjeux théoriques sont de taille, semblables à ceux qui hantent l'étude des concepts politiques à travers l'histoire occidentale (Richter 1995): un exemple parmi légions, ce que l'on entend par «bourgeoisie" à l'époque de la Renaissance est très différent de l'objet signifié par ce même terme au $\mathrm{XIX}^{\mathrm{e}}$ siècle. L'étude sur la longue période constitue indéniablement un terrain miné. Néanmoins, il y a urgence de récupérer — sur ce sujet comme sur tant d'autres - une perspective plus large. Et c'est donc en portant sur la matière un regard plus inclusif qu'il a été envisagé d'avancer un peu plus loin, en tentant une comparaison développée non seulement sur l'axe du temps mais sur celui de l'espace aussi. Il a semblé utile d'interroger au moins deux univers non chrétiens, dans lesquels le discernement n'est pas issu du paradigme paulinien et n'est pas désigné par une terminologie qui nous est familière, c'est-à-dire issue du grec (diakrisis) ou du latin (discre- 
tio). Pourquoi ce choix? Pour souligner l'importance de deux questions centrales dans la compréhension des faits humains. En premier lieu, il y a la question des emprunts culturels, caractéristique d'une approche de type diffusionniste: est-il possible, par exemple, de repérer des éléments du discernement chrétien dans la tradition juive précédente, plus particulièrement celle qui est représentée à Qumran (Duhaime) ? En deuxième lieu, se pose la question des analogies sans contacts culturels apparents: la dichotomie vrai-faux ou bon-mauvais dans le domaine du spirituel, qui imprègne l'axiologie humaine et inspire donc l'agir du décideur, est-elle un fait universel, structural (au sens du structuralisme) et propre à l'être humain indépendamment de son milieu culturel ? Cette question, qui est celle de l'universalisme, justifie une incursion dans la Chine ancienne, monde culturel où l'on ne penserait peut-être pas retrouver une telle problématique (Ghiglione).

La comparaison est nécessaire à la connaissance: c'est un constat dont on ne peut faire l'économie (Boesflug-Dunand 1997, Paden 2005). Or, malgré sa mauvaise presse dans bien des milieux en sciences humaines - et ce, principalement en raison de son utilisation ethnocentrique (pour ne pas dire impérialiste: Smith 1978; 1990) à l'époque coloniale —, elle demeure une démarche incontournable pour toute étude qui veuille s'élever au-dessus du particularisme descriptif. Il faut reprendre en main le problème des catégories universelles et briser l'équivalence - non injustifiée mais quelque peu expéditive - que les études postcoloniales ont établie avec l'impérialisme culturel (Paden 2000). L'entreprise est risquée et requiert des précautions ainsi qu'une profonde lucidité méthodologique: on invoquera à notre défense, ici, le caractère tout à fait expérimental et provisoire de cette tentative. Elle se veut, à ce stade, un déclencheur de réflexion. Il sera donc utile, dans les lignes qui suivent, de commenter les différentes contributions qui ont été accueillies dans ce numéro afin de souligner de quelle manière et dans quelle mesure elles ont tenté de répondre à la question sur la nature, le rôle et la fonction du discernement spirituel dans la religion.

Le texte de Jean Duhaime sur Qumrân alimente la réflexion autour du problème délicat des antécédents du charisme paulinien. Il est intéressant de constater que dans ce milieu sectaire, qui présente de nombreuses analogies non seulement avec les premières communautés chrétiennes mais plus encore avec le monachisme des origines, la reconnaissance d'un savoir surnaturel sert à différencier les élus des égarés, en d'autres termes "nous » et les «autres». Faute de pouvoir retracer dans ce corpus une véritable 
théorie du discernement, il est possible à tout le moins de constater — ce qui n'est pas un gain interprétatif négligeable - que l'accès direct à une connaissance supérieure constitue une prérogative des figures notables de la communauté d'élection, en l'espèce le Maître de Justice et le Maskil. C'est une situation qui, sans compter les exemples de Jésus ou de Montan ${ }^{1}$, se présentera à nouveau aux $\mathrm{III}^{\mathrm{e}}$ et $\mathrm{IV}^{\mathrm{e}}$ siècles dans les communautés manichéennes et pachômiennes (Vecoli 2011). Ce constat, en ce qui concerne Qumrân, offre à l'historien deux pistes exégétiques, qui ne sont pas nécessairement contradictoires. D'une part, la reconnaissance d'une science surhumaine à deux personnalités importantes de la communauté met en évidence la fonction de légitimation de l'autorité qui est propre au "discernement "; ce dernier se configure alors comme le fondement du charisme au sens wébérien du terme. D'autre part, une lecture typologique de la figure du chef - considéré comme idéaltype de l'adepte de la communauté - induit à considérer la connaissance exclusive qu'il détient comme la preuve de l'élection du groupe par rapport au monde social environnant. Le discernement est par suite l'instrument d'affirmation d'une identité d'exception dans un contexte conflictuel. Ainsi, il justifie ce que le sociologue Roy Wallis appelait le «rejet du monde» (Wallis 1984): grâce à ce don divin, il est possible d'affirmer que "nous " savons et que les «autres» ignorent ou se trompent. L'intervention de Duhaime permet donc d'apprécier la continuité - sur la longue période et à travers deux traditions religieuses différentes et pourtant reliées (judaïsme et christianisme) - des fonctions socioreligieuses associées à la prétention de posséder en exclusivité le regard du ciel sur les choses de la terre. Dans la dichotomie radicale posée par ce regard ultramondain, on reconnaît une dimension polémique qui revient dans d'autres contextes. Reste à savoir si les ressemblances que l'on croit déceler dans les variations de l'histoire religieuse constituent des récurrences explicables sur le plan sociologique ou imputables à des emprunts culturels: avons-nous affaire à des analogies ou à des généalogies? Les pages sur Qumran permettent de poser sérieusement la question et d'affirmer par conséquent la légitimité d'une comparaison.

La contribution d'Anna Ghiglione sur la critique moïste de la guerre offensive confirme la récurrence de la notion de discernement dans la tradition chinoise. La perspicacité dont il est question ici relève d'un raison-

1. Il semble opportun, pour le moins à ce stade et malgré toutes les superpositions qu'il est possible d'établir, de considérer à part les phénomènes plus spécifiquement prophétiques. 
nement dialectique qui procède par différentiations de couples d'opposés inconciliables: la connaissance résulte de la capacité à reproduire dans la variabilité des situations le même processus - tenu pour valable - de dégagement de la vérité et d'organisation ordonnée du réel. Si une vérité vaut dans certaines conditions, on doit reconnaître - et c'est à ce moment qu'apparaît le discernement - qu'elle doit valoir dans des conditions apparemment différentes mais dans les faits analogues. Si l'agression immotivée d'un individu est condamnable, il en découle que la guerre offensive doit l'être également, car cette dernière partage les caractéristiques prégnantes qui définissent la première: seule l'échelle change. Ceux qui ne le comprennent pas manquent de discernement. En effet, il n'est pas donné à tous de reconnaître l'équivalence, car celle-ci est mise en évidence par un certain type de raisonnement. L'intervention de Ghiglione remet en perspective le stéréotype récurrent d'une culture chinoise toute axée sur l'analogie corrélative et sur l'holisme par la conciliation des contraires. Et pourtant, même dans une Chine imprégnée par la tradition taoïste, on ressent l'exigence de l'affirmation d'une vérité exclusive, non sujette à une relativisation herméneutique mais défendue comme une découverte qui éclaire. Ce qu'il reste à vérifier, dans ce contexte comme dans tout autre milieu non chrétien, c'est l'impact dans la sphère plus proprement religieuse d'un discernement philosophique (bien conscient que nous sommes du caractère culturellement connoté, donc problématique, de la différentiation des concepts de philosophie et de religion). Pour l'instant, l'intervention de Ghiglione nous montre l'essor d'une méthode dialectique dont la sphère d'application s'étend particulièrement à la réflexion éthique. La question d'un discernement surnaturel, entendu comme connaissance fondée dans la dimension métaphysique du réel, demeure ouverte et méritera certainement d'être traitée ultérieurement.

Le discernement dans la théorie monastique ancienne - objet de ma contribution - ainsi que celui décrit par Ignace de Loyola dans les Exercices spirituels - dont il est question dans le texte de Guido Mongini - constituent deux cas centraux, souvent élevés en véritables paradigmes pour la théologie chrétienne. S'il est vrai que l'importance de la spécificité du contexte historique ne peut être sous-estimée, la récurrence d'un certain nombre d'éléments clés dans ce que l'on pourrait nommer la "phénoménologie » (au sens rigoureusement étymologique du terme) du discernement justifie une interrogation plus poussée sur la question de la longue durée. En effet, il ressort clairement que le discernement est dans les deux cas en relation avec le problème de la décision, qu'il fonde dans un ordre méta- 
physique et qu'il présente - même dans ses fondements spirituels comme strictement dichotomique. On remarquera également que, dans la littérature monastique ancienne comme dans les œuvres d'Ignace, on entend réactiver l'image eschatologique du champ de bataille où les armées de la lumière et celles des ténèbres s'affrontent pour l'âme humaine (anges et démons du désert dans la littérature des Apophtegmes, les deux étendards dans les Exercices spirituels). De plus, dans ces deux lieux de l'histoire chrétienne, le discernement se situe — non sans ambigüités — à la frontière entre technique humaine et charisme divin. D'une part, l'irruption de l'Esprit constitue toujours un élément irréductible et autoévident de l'expérience religieuse, véritable socle axiomatique de toute appréciation ultérieure des évolutions ou involutions de la vie spirituelle. D'autre part, les sources s'engagent systématiquement dans l'élaboration d'un savoir "empirique" psychologique fondé sur l'expérience. Il s'agit d'une capacité d'analyse et de distinction des répercussions mentales produites par la manifestation des différentes inspirations vouées à orienter l'action. Ce ne sont que quelques exemples, mais ils sont suffisants pour poser la question: la continuité découle-t-elle d'une diffusion par transmission culturelle à travers les siècles d'une récursivité attribuable à la "génétique » même du christianisme, ou d'une fonctionnalité structurelle - pour ne pas dire cognitive - de l'être humain? Faut-il réellement choisir entre ces différentes pistes explicatives ou y a-t-il moyen de les concilier?

Il convient ici de noter que, dans l'hypothèse d'une généalogie culturelle, pèse l'absence, dans ce numéro, d'une intervention sur la période médiévale: la théorie du discernement y connaît un développement essentiel, provoqué par une croissante inquiétude de l'Église institutionnelle face à l'effervescence du phénomène mystique; si le sujet n'a pas manqué d'intéresser les historiens du christianisme (Elliott 2002, Anderson 2011), il requiert encore à ce jour d'être resitué sur la période longue de cette tradition religieuse.

Le regard anthropologique de Géraldine Mossière et de Guillaume Boucher nous introduit à la réalité du discernement spirituel dans un choix de communautés religieuses contemporaines, pour la plupart appartenant à la constellation vaste et complexe des mouvements dits d'effusion de l'Esprit. Dans les terrains d'enquête sélectionnés, on trouve confirmation de la double nature de cette connaissance supérieure, à la fois charismatique et technique, car si elle dépend certes d'une effusion de la libéralité divine, elle se concrétise néanmoins dans les formes codifiées d'un examen détaillé de la psychologie individuelle. Cette ambivalence, déjà soulignée 
dans les interventions précédentes (et qu'il est intéressant de confirmer dans ce contexte), explique le fait que le discernement puisse être, selon les cas, instrument objectif de contrôle de manifestations spirituelles potentiellement subversives ou signe de reconnaissance d'un maître charismatique (au sens wébérien du terme) à l'intérieur de communautés peu ou pas institutionnalisées. Dans les deux volets de l'alternative, il constitue l'instrument de légitimation de l'autorité religieuse. Le caractère contraignant de cette autorité se donne à voir dans son exercice sur l'intériorité des croyants: les témoignages recueillis confirment une donnée récurrente de l'histoire chrétienne, à savoir que le discernement agit sur la subjectivité individuelle par l'institution d'un véritable tribunal de la conscience. Ce fait favorise ou reflète (difficile de trancher) une individualisation de la relation au divin ainsi que du processus décisionnel humain. S'il est vrai que les manifestations contemporaines de ce pouvoir cognitif métaphysique s'accordent bien avec une insistance typiquement postmoderne de recentralisation sur le soi, en tant qu'herméneutique de l'intériorité, elles confirment néanmoins sur d'autres fronts un caractère résolument disciplinaire, voire autoritaire, qui date sans doute de son utilisation médiévale comme instrument de contrôle de la mystique. Bien qu'arbitraire dans les modalités de son exercice (particulièrement quand il est activé dans la pratique de la direction spirituelle), le discernement ne s'inscrit nullement dans une dynamique de relativisation postmoderne de la vie spirituelle individuelle. En effet, il vise plutôt à départager selon une binarité axiologique non négociable chaque mouvement de l'esprit. Concrètement, au niveau communautaire, il représente le chien de garde qui empêche les mouvements charismatiques de se liquéfier dans le chaos d'une vie de l'Esprit non balisée. Si l'intervention de Mossière-Boucher ouvre à une comparaison extra-chrétienne, par une incursion dans une communauté interreligieuse, cela constitue une incitation à vérifier dans d'autres contextes, plus éloignés du monde occidental, l'ensemble des fonctions sociales attribuées au discernement: est-ce un concept susceptible de traduction et donc de translation dans des milieux religieux radicalement différents? Et si oui, dans quelle mesure?

Enfin, la réflexion d'Ignace Ndongala Maduku à propos du discernement dans l'œuvre de trois théologiens catholiques (M.-D. Chenu, J.-M. R. Tillard et C. Théobald) témoigne de l'importance de ce charisme pour la vie de l'Église contemporaine. Par sa mise en relation avec les « signes des temps » qu'il est sensé révéler, ce don spirituel revêt les formes d'une science inductive de déchiffrement de l'histoire humaine selon une 
perspective de foi. Les exigences de plus en plus pressantes d'une adaptation de l'Église à la modernité occidentale (qui aboutiront à l'aggiornamento du Concile Vatican II) signalent l'urgence d'harmoniser la doctrine chrétienne à l'époque dans laquelle elle est proclamée. Pour ce faire, il s'avère nécessaire de prendre en compte les transformations de l'histoire et de les interpréter comme données parlantes sur un plan spirituel. Cette approche présente de nombreuses et frappantes analogies avec l'empirisme sapientiel vétérotestamentaire, qui pointe le regard sur la création afin d'y déceler les signes - indirects et cependant visibles pour ceux qui savent voir - du dessein de Dieu. À l'homme revient, par le biais du discernement, la responsabilité de décliner la doctrine selon les contingences contextuelles du temps. Comme dans la direction spirituelle, le discernement devient le dispositif d'adaptation aux cas particuliers de la norme universelle. En même temps, il rejoint une fonction exercée dans bien d'autres dimensions de la vie religieuse, celle de fournir un encadrement à la libéralité interprétative des croyants. En ce sens, il définit le lieu où se gère la tension alimentée par les incohérences d'une communauté religieuse. À l'intérieur de l'Église, il sert le magistère dans son œuvre de filtrage des inspirations venant de l'assemblée des fidèles: il y a lieu de souligner que cette fonction représente une extension sur le plan ecclésial de son rôle original de triage - pour ainsi dire — des esprits qui se manifestent dans l'intériorité du croyant.

À la fin de ce parcours, il ne sied pas d'offrir aux lecteurs des réponses définitives sur la place du discernement dans la vie religieuse. En revanche, il convient de constater que, malgré la spécificité des contextes socioculturels examinés et des disciplines déployées dans l'analyse, maintes analogies sont apparues en ce qui a trait aux modalités d'apparition/reconnaissance et aux fonctions sociopolitiques de ce surplus cognitif, toujours revendiqué au nom d'un ordre métaphysique. Il apparaît très clairement que le discernement est le lieu de traduction en praxis de l'épistémologie religieuse. Il s'agit d'un concept névralgique dans la relation toujours délicate entre croyance et action, et de ce fait il est un facteur clé de la vie religieuse occidentale. Il est donc souhaitable que le présent dossier inspire un approfondissement dans l'étude de cet objet mystérieux. 


\section{Références}

Anderson, W. L. (2011), The Discernment of Spirits. Assessing Visions and Visionaries in the Late Middle Ages, Tübingen, Mohr Siebeck.

Assmann, J. (1997), Moses the Egyptian. The Memory of Egypt in Western Monotheism, Cambridge, Harvard University Press.

Boesflug, F. et Dunand, F. (1997), dir., Le comparatisme en histoire des religions, Paris, Cerf, p. 7.

Copeland, C. et Machielsen, J. (2013), dir., Angels of Light? Sanctity and the Discernment of Spirits in the Early Modern Period, Leiden/ Boston, Brill.

Elliot, D. (2002), "Seeing Double. John Gerson, the Discernment of Spirits, and Joan of Arc ", American Historical Review, 107, p. 26-54.

Paden, W. E. (2000), "Elements of a New Comparativism", dans K.C. Patton et B. C. Ray, dir., A Magic Still Dwells Comparative Religion in the Postmodern Age, Berkeley/Los Angeles, University of California Press, p. 182-192.

(2005), "Comparative Religion", dans J. R. Hinnels, dir., The Routledge Companion to the Study of Religion, London/New York, Routledge, p. 208-225.

Rich, A. D. (2007), Discernment in the Desert Fathers. Diakrisis in the Life and Thought of Early Egyptian Monasticism, Milton Keynes, Paternoster.

Richter, M. (1995), The History of political and social Concepts. A Critical Introduction, New York, Oxford University Press.

SMith, J. Z. (1978), "Map is not Territory ", Map is not Territory. Studies in the History of Religions, Leiden, Brill, p. 289-309.

(1990), Drudgery Divine. On the Comparison of Early Christianities and the Religions of Late Antiquity, Chicago, University of Chicago Press.

Vecoli, F. (2006), Lo spirito soffia nel deserto. Carismi, discernimento e autorità nel monachesimo egiziano antico, Brescia, Morcelliana.

(2011), "Communautés religieuses dans l'Égypte du IV e siècle: manichéens et cénobites ", Historia Religionum, 3, p. 23-46.

Wallis, R. (1984), The Elementary Forms of the New Religious Life, London, Routledge and Kegan Paul. 